\title{
TRANSICIÓN DE MADERA JUVENIL A MADURA EN UN CLON DE Populus deltoides IMPLANTADO EN BUENOS AIRES ARGENTINA
}

\author{
JUVENILE TO MATURE WOOD TRANSITION IN ONE CLONE OF \\ Populus deltoides IMPLANTED IN BUENOS AIRES, ARGENTINE
}

\author{
Ana C. Cobas ${ }^{1,3}$, María C. Area ${ }^{2,3}$, Silvia Monteoliva ${ }^{1,34}$
}

\begin{abstract}
RESUMEN
El objetivo del trabajo fue determinar la edad de transición de madera juvenil a madura a partir de las propiedades de la madera en un clon de álamo. Se muestrearon cinco árboles de 17 años de Populus deltoides "Australia 129/60" de una plantación comercial. Se tomaron muestras a cinco alturas en el fuste: $0,3 \mathrm{~m}, 1,3 \mathrm{~m}, 4,2 \mathrm{~m}, 8,1 \mathrm{~m}$ y $15,9 \mathrm{~m}$ y se determinó anualmente: crecimiento, densidad de la madera, morfometría de fibras y vasos y área de pared. La edad de transición de madera juvenil a madura se determinó por el método de regresión segmentada. Los patrones de variación radial y axial de las diferentes propiedades fueron de un aumento de sus magnitudes con la edad y altura en el fuste. La edad de transición entre madera juvenil y madera madura no fue idéntica para todas las propiedades, definiéndose edades de transición de 4, 5, 7 y 9 años dependiendo de la variable. La secuencia de maduración fue: área de pared, diámetro y frecuencia de vasos, ancho y longitud de fibras, densidad y espesor de pared. Las propiedades de la madera juvenil fueron inferiores con respecto a la madura, siendo el volumen del cilindro juvenil del 52\%.
\end{abstract}

Palabras clave: Variación radial, regresiones segmentadas, morfometría de fibras, vasos, madera juvenil.

\section{ABSTRACT}

The aim of this work was to determine the age at which the transition of the juvenile wood to the mature wood takes place, by means of the parameters: density, morphometry of fibers, and vessels in one poplar clone. Five 17-year-old Populus deltoides "Australia 129-60" trees were sampled from a commercial plantation in Buenos Aires, Argentina. Samples were taken at 5 heights from the trunk: 0.3 $\mathrm{m}, 1.3 \mathrm{~m}, 4.2 \mathrm{~m}, 8.1 \mathrm{~m}$ and $15.9 \mathrm{~m}$ and the annual radial growth, wood density, morphometry of fibers and vessels, and cell wall area were measured. Segmented regression analyses were used to determine the age of transition from juvenile to mature wood. The radial variation of different anatomical properties increased with age. The estimated age of transition from juvenile wood and mature wood was not identical for all properties. The transition ages were 4, 5, 7 and 9 years depending on the variable. The sequence of maturation was: cell wall area, vessel diameter and frequency, fiber width and length, density and wall thickness. Juvenile wood properties were significantly lower with respect to the mature wood. The volume of the juvenile wood was $52 \%$.

Keywords: Radial variations, segmented regression, fibre properties, vessels, juvenile wood.

\footnotetext{
${ }^{1}$ Facultad de Ciencias Agrarias y Forestales, Universidad Nacional de La Plata. Av. 60 y 119. CC 31 (1900) La Plata, Argentina.

${ }^{2}$ Programa de Celulosa y Papel, Facultad de Ciencias Exactas, Químicas y Naturales, Universidad Nacional de Misiones,

Félix de Azara 1552 (3300) Posadas, Misiones, Argentina.

${ }^{3}$ Consejo Nacional de Investigaciones Científicas y Técnicas (CONICET), Buenos Aires, Argentina

Autor para correspondencia: smonteoliva@yahoo.com.ar

Recibido: 31.08.2012 Aceptado: 15.10.2012
} 


\section{INTRODUCCIÓN}

Los valores de las propiedades de la madera están influenciados por la posición dentro del árbol. Se han demostrado patrones de variación radial (médula- corteza) y patrones de variación axial (baseápice) en diferentes especies, asociados a distintas edades fisiológicas del cámbium y por lo tanto a la formación de madera juvenil y madura (Matyas y Peszlen 1997, Zobel y Sprague 1998, DeBell et al. 2002). El leño juvenil fue definido para las coníferas como la zona más cercana al centro del árbol que se extiende desde la base hasta el ápice. El tamaño de este cilindro central puede variar considerablemente entre árboles de una misma especie y edad (Zobel y Jett 1995) y también dependiendo de la propiedad que se utilice para su delimitación (Tasissa y Burkhart 1998, Bhat et al. 2001). La literatura indica que la madera juvenil está asociada a una edad fisiológica temprana de las células cambiales y presenta menor densidad, elementos fibrosos más cortos y propiedades de resistencia menores en comparación con la madera madura (Zobel y Sprague 1998, DeBell et al. 2002).

Se han publicado algunos trabajos de álamos donde se determina la edad de transición entre madera juvenil y madura para diferentes especies, sitios y edades $\mathrm{y}$, a su vez, a través de diferentes métodos de análisis. Bendtsen y Senft (1986) determinaron que el punto de demarcación entre madera juvenil y madura en Populus deltoides de 30 años fue de 17-19 años para densidad, longitud de fibras y ángulo microfibrillar, calculados a través de regresión lineal mediante la iteración de pendientes sucesivas. Matyas y Peszlen (1997) cuantificaron la edad de transición entre los leños por el método de regresión lineal segmentada, en 3 clones de $P$. $x$ euroamericana y para siete variables anatómicas, demostrando que la edad de transición varía de acuerdo a la propiedad analizada y al sitio; sin embargo el orden de maduración no cambia.

La producción de Salicáceas (Populus spp., Salix spp.) se conforma como el tercer cultivo en importancia en la Argentina, después de los pinos y eucaliptos (SAGPyA Forestal 2004). La región más importante para la producción de salicáceas se ubica en el Bajo Delta del Paraná y alcanzan una superficie cercana a 58.000 ha. (Borodowski 2006). En el caso particular del álamo, la producción está orientada a abastecer a los aserraderos de la zona y a la producción de paneles de compensados (madera laminada). La porción remanente es generalmente aplicada a la producción de tableros de partículas, embalajes industriales de tipo variado (como pallets y cajones para aplicación fruti-hortícola) y pulpa para papel de diario (Borodowski 2006). Las características de las plantaciones, clones utilizados, tratamiento silvícola y turno de corte dependen del producto final industrial. En la composición actual de las plantaciones comerciales de álamo predominan los clones de Populus deltoides, seguidos de clones de $P$ x canadensis (Achinelli 2006). El P. deltoides 'Australia 129/60' es uno de los clones que mas se cultivan actualmente tanto en el Delta como en tierra firme (Provincia de Buenos Aires) y su destino industrial es fundamentalmente el aserrado y laminado, con turnos de rotación de entre 15 y 22 años.

Para algunos clones de álamos implantados en Argentina, se han descrito variaciones de las propiedades de la madera ligadas a los clones y sitios (Monteoliva y Senisterra 2008), pero no se ha generado información que cuantifique a través de distintas variables de la madera la transición entre madera juvenil y madura.

La determinación de la edad de transición entre leños permitiría calcular el volumen de madera juvenil al turno. El orden de maduración de los elementos celulares en forma diferencial permitiría seleccionar la edad límite según el destino industrial de la misma (aserrado o triturado). Esta información podría también ser de utilidad para definir un manejo silvícola que tienda a mejorar la calidad o disminuir el volumen del cilindro central juvenil.

El objetivo del trabajo fue determinar la edad de transición de madera juvenil a madura a partir de la densidad, morfometría de fibras y vasos y área de pared en Populus deltoides "Australia 129-60". 


\section{MATERIALES Y MÉTODOS}

Se estudiaron árboles de una plantación comercial de Populus deltoides "Australia 129-60" procedentes de la $4^{\circ}$ Sección de Islas del Delta, en la Provincia de Buenos Aires, Argentina (34 $30^{\circ}$ Lat. Sur, $59^{\circ} 00^{\prime}$ Long. Oeste). La plantación, con destino final de laminado, se implantó con un distanciamiento de $5 \mathrm{~m} \mathrm{x} 4 \mathrm{~m}$. Se utilizó un sistema de monte alto, efectuándose dos podas hasta los 7 $\mathrm{m}$ de altura. El turno de corta está definido entre los 15 y 22 años.

Se seleccionaron cinco árboles con buen estado sanitario de 17 años de edad y con las características epidométricas que se indican en la tabla 1.

Tabla 1. Características de los árboles muestreados

\begin{tabular}{cccc}
\hline Árbol & $\begin{array}{c}\text { Altura } \\
\text { comercial } \\
(\mathrm{m})\end{array}$ & $\begin{array}{c}\text { DAP s/c } \\
(\mathrm{cm})\end{array}$ & $\begin{array}{c}\text { Volumen } \\
\left(\mathrm{m}^{3}\right)\end{array}$ \\
\hline 1 & 25,20 & 46,70 & 1,70 \\
2 & 24,01 & 34,71 & 1,04 \\
3 & 25,70 & 40,03 & 1,37 \\
4 & 24,03 & 38,05 & 1,11 \\
5 & 24,40 & 27,70 & 0,67 \\
\hline
\end{tabular}

Altura comercial: altura hasta los $7 \mathrm{~cm}$ de diámetro, DAP s/c: diámetro a la altura de pecho sin corteza

Se apearon y cortaron rodelas ( $5 \mathrm{~cm}$ de espesor) a cinco alturas en el fuste: $0,3 \mathrm{~m} ; 1,3 \mathrm{~m} ; 4,2 \mathrm{~m} ; 8,1$ $\mathrm{m}$ y $15,9 \mathrm{~m}$ del suelo. De cada rodela se sacó una tabla central de $3 \mathrm{~cm}$ de ancho en la cual se marcaron los anillos de crecimiento, previa preparación de la superficie transversal con sucesivas lijas de diferentes granulometrías hasta la perfecta visualización de los elementos celulares. Para la delimitación y medición de los anillos se utilizó una lupa binocular con reglilla $(10 \mathrm{x})$. Sobre la tabla se cortaron probetas en cada anillo de crecimiento abarcando la totalidad del ancho del anillo, en ambos radios. Por lo tanto, en cada altura de muestreo se obtuvieron 2 muestras por anillo para determinación de densidad y morfometría celular (duplicado), cuyos valores se promediaron. Además de obtuvo una astilla por probeta para realizar macerados y medir la longitud de fibras.

El análisis de las variaciones en el fuste se realizó radial y axialmente. La variación radial se analizó para todas las propiedades a una única altura en el fuste $(1,3 \mathrm{~m})$ y la variación axial solo en dos propiedades muy importantes consideradas como índices de calidad en la industria papelera (longitud de fibras) y en la industria del aserrado (densidad de la madera).

La determinación de densidad básica $\left(\mathrm{kg} \mathrm{m}^{-3}\right)$ se realizó según la norma IRAM 9544 (Instituto Argentino de Normalización 1973). Se registró el volumen saturado por inmersión total de la pieza en agua, y el peso anhidro en estufa a $102{ }^{\circ} \mathrm{C}+/-3{ }^{\circ} \mathrm{C}$, pesado inmediatamente después de que la pieza alcanzara un equilibrio térmico con el ambiente. La densidad se determinó por el cociente entre el peso anhidro de la muestra y el volumen saturado de la misma.

Para la determinación de la longitud de fibras $(\mathrm{mm})$ se midieron 60 fibras por edad cambial (anillo de crecimiento) por árbol sobre imágenes digitales tomadas con un microscopio óptico, previa maceración de dichas posiciones según la técnica de maceración de Franklin (1945) (total mediciones por edad y altura de muestreo $n=300$ ). 
Las mediciones de ancho de fibras $(\mathrm{mm}, \mathrm{n}=300)$, espesor de pared $(\mathrm{mm}, \mathrm{n}=300)$, diámetro de vasos ( $\mathrm{mm}, \mathrm{n}=500)$, frecuencia de vasos $\left(\mathrm{n} / \mathrm{mm}^{2}, \mathrm{n}=50\right.$ campos) y proporción de pared $(\%, \mathrm{n}=50$ campos) se realizaron sobre imágenes digitales de microscopía óptica de cortes histológicos transversales teñidos con safranina en cada anillo de crecimiento (por duplicado) de una única altura en el fuste $(1,3 \mathrm{~m})$.

Se realizaron ANOVAS con edad cambial, árbol, altura de muestreo y con las zonas de madera juvenil y madera madura, como fuentes de variación. El contraste de medias se realizó con la prueba de Tukey $(\mathrm{p}<0,05)$.

Para determinar la edad de transición de madera juvenil a madura, se utilizó el método de regresión segmentada sobre el patrón radial de la propiedad a analizar. Este método supone que en el patrón radial de la misma (asociado con la edad del anillo de crecimiento) existe un cambio notorio en la pendiente de la línea de regresión de la propiedad en estudio correspondiente a la edad de transición. El modelo de regresión por segmentos general se presenta en la ecuación 1 y los modelos para las porciones de madera juvenil y madura en las ecuaciones 2 y 3 respectivamente (Tasissa y Burkhart 1998).

$$
\mathrm{Yi}=\beta_{0}+\beta_{1} \mathrm{x}_{\mathrm{i}}+\beta_{2}\left(\mathrm{x}_{\mathrm{i}}-\mathrm{T}\right) \mathrm{x}_{2}+\mathrm{e}_{\mathrm{i}}
$$

Donde:

$\mathrm{Yi}=$ característica de interés en el i-ésimo anillo de crecimiento

$\beta_{0}, \beta_{1}, \beta_{2}=$ coeficientes de regresión

$\mathrm{x}_{\mathrm{i}}=$ edad de formación del i-ésimo anillo de crecimiento

$\mathrm{T}=$ edad de transición de madera juvenil a madura

$\mathrm{x}_{2}=$ variable indicadora : $\mathrm{x}_{2}=0$ si $\mathrm{x}_{\mathrm{i}}<\mathrm{T}$ (madera juvenil) o $\mathrm{x}_{2}=1$ si $\mathrm{x}_{\mathrm{i}}>\mathrm{T}$ (madera madura)

$\mathrm{e}_{\mathrm{i}}=$ error aleatorio asociado al i-ésimo anillo de crecimiento.

De esta manera cuando $\mathrm{x}_{2}=0$ (madera juvenil):

$$
\mathrm{E}(\mathrm{Yi})=\beta_{0}+\beta_{1} \mathrm{x}_{\mathrm{i}}
$$

y cuando $\mathrm{x}_{2}=1$ (madera madura)

$$
\mathrm{E}(\mathrm{Yi})=\beta_{0}-\left(\beta_{2} \mathrm{~T}\right)+\left(\beta_{1}+\beta_{2}\right) \mathrm{x}_{\mathrm{i}}
$$

Se aplicó el método Piecewise Linear Regression (Breakpoint Regresión, Statistica v6).

\section{RESULTADOS Y DISCUSIÓN}

\section{Variación radial de las propiedades}

Los análisis de la varianza indicaron que el árbol no resultó una fuente significativa de variación para las propiedades de la madera ( $p>0,05)$, excepto para densidad básica $(p<0,05)$. La edad cambial y la altura de muestreo en el fuste, por otro lado, influyeron significativamente $(p<0,05)$ sobre todas las propiedades de la madera y el crecimiento anual.

Se determinó, a la altura de 1,3 m en el fuste, un patrón general de aumento de la magnitud de las variables con la edad, con diferentes fluctuaciones en las edades intermedias. La frecuencia de vasos tuvo un comportamiento inverso, disminuyendo con la edad (Figura 1). 

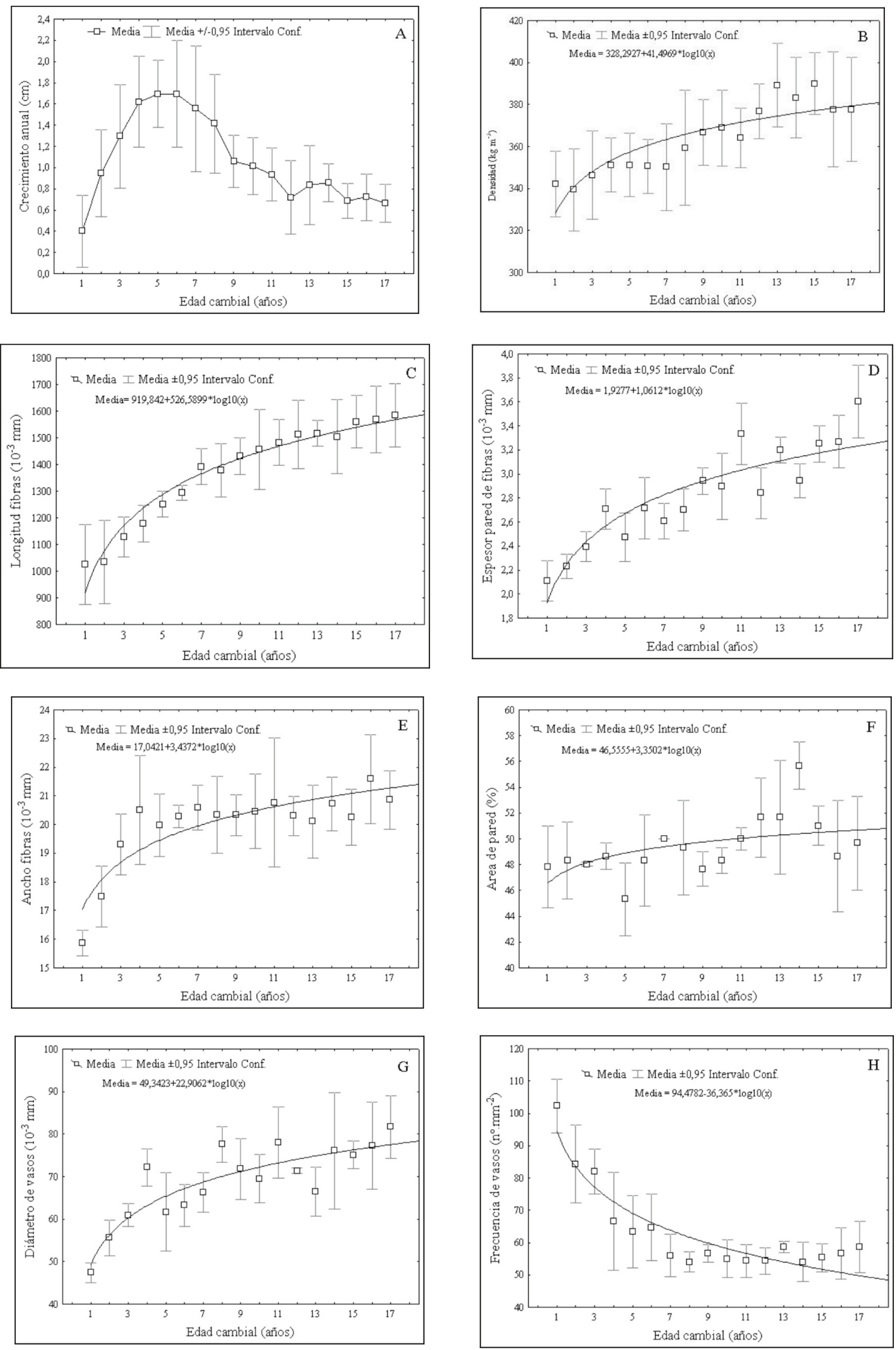

Figura 1. Patrones de variación radial (anual) de las variables estudiadas a la altura de 1,3 $\mathrm{m}$. Línea de tendencia con un ajuste exponencial. 
Los valores de crecimientos anuales (CA), medidos como ancho de anillo, tuvieron un aumento rápido hasta llegar a un máximo en los años 4,5 y 6 . A partir del año 7 los crecimientos comienzan a disminuir (Figura 1 A). La densidad de la madera presentó un suave aumento de sus valores a medida que se incrementa la edad cambial, encontrándose diferencias significativas sólo entre los primeros dos anillos y los años 13 a 17 según la prueba de Tukey (Figura 1 B). La longitud de fibra y espesor de pared presentaron un rápido aumento de sus valores hasta la edad de 6-7 años. A partir de esa edad, los valores de longitud se incrementan gradualmente pero sin presentar diferencias significativas entre sí, mientras que los de espesor de pared fluctúan alrededor de un valor medio y presentando los mayores valores en los años 11 y 17 (Figura 1 C y D). Los valores de ancho de fibras presentaron un aumento sostenido hasta los 4 años y luego se estabilizaron sin diferencias significativas entre sus valores medios (Figura $1 \mathrm{E})$. El área de pared no presentó grandes variaciones con la edad, siendo el año 5 el de menor valor y el año 14 el mayor (Figura $1 \mathrm{~F}$ ). Los vasos presentaron un rápido y significativo aumento de los valores de diámetros medios hasta los 4 años, acompañado por una fuerte caída de su frecuencia; a partir del año 5 los valores de diámetro y frecuencia de vasos fluctúan alrededor de un valor medio (Figura $1 \mathrm{G} \mathrm{y} \mathrm{H)}$.

Para las variables densidad y longitud de fibras se realizó el análisis de los patrones radiales en 5 alturas del fuste (Figura 2). Se determinó que los patrones radiales descritos a la altura de 1,3 $\mathrm{m}$ se repitieron a lo largo del fuste.

Los patrones radiales de variación de los valores de las diferentes propiedades presentan un aumento con la edad (excepto en el caso de la frecuencia de vasos) coincidentes con la bibliografía (Matyas y Peszlen 1997, DeBell et al. 2002). 


\section{Variación axial}

Según el ANOVA, la altura de muestreo en el fuste fue una fuente significativa de variación para la densidad y longitud de fibras $(\mathrm{p}<0,05)$. La densidad aumentó con la altura de muestreo presentando diferencias significativas solo en las 2 alturas superiores $(8,1 \mathrm{~m}$ y $15,9 \mathrm{~m})$. Para la longitud de fibras la tendencia axial fue de un leve aumento hasta las alturas intermedias y luego un descenso. El patrón de la longitud de fibras muestra una menor variabilidad que el de densidad (Figura 2).
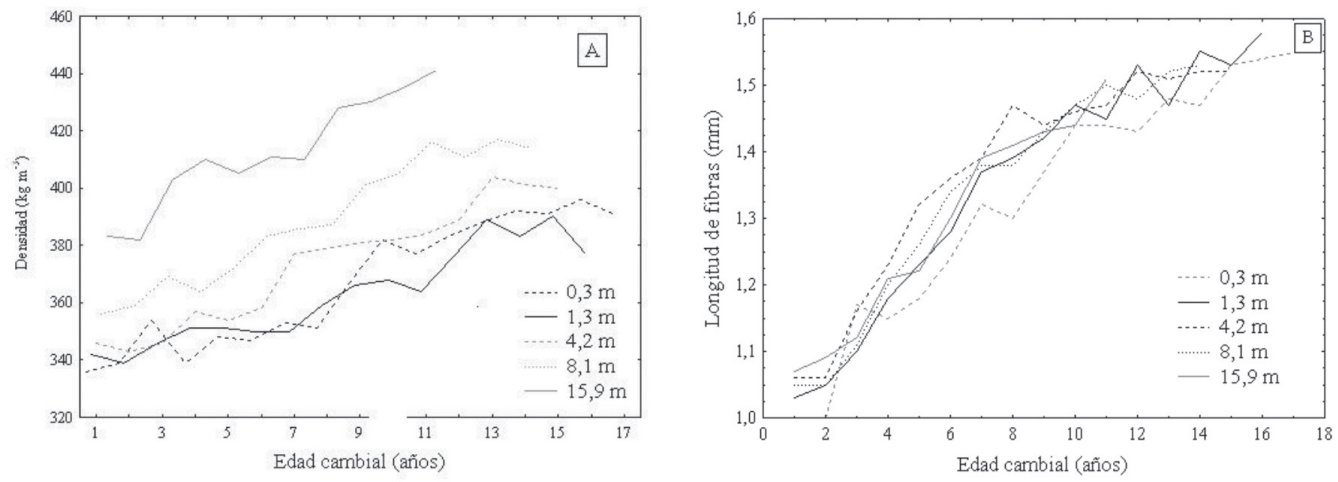

Figura 2. Patrones de variación radial de la densidad y longitud de fibras en las diferentes alturas de muestreo en el fuste.

\section{Edad de transición entre madera juvenil y madura}

$\mathrm{Al}$ aplicar el modelo de segmentación lineal se obtuvieron las ecuaciones de regresión que figuran en la tabla 2 para la altura de $1,3 \mathrm{~m}$. La edad estimada de transición entre madera juvenil y madura no fue idéntica para todas las propiedades. La secuencia de maduración de los parámetros fue: diámetro de vasos, área de pared, frecuencia de vasos, ancho de fibras, longitud de fibras, densidad y espesor de pared de las fibras.

Tabla 2. Edad de transición entre madera juvenil y madura para las variables estudiadas a la altura de 1,3 m; ecuaciones de ajuste, valor de $\mathrm{R}$ y valor $\mathrm{p}$

\begin{tabular}{lcllll}
\hline $\begin{array}{c}\text { Variable } \\
\text { dependiente }\end{array}$ & $\begin{array}{c}\text { Edad de } \\
\text { transición } \\
(\text { años })\end{array}$ & $\begin{array}{l}\text { Tipo de } \\
\text { madera }\end{array}$ & $\begin{array}{c}\text { Modelos } \\
\mathrm{y}=\text { ar. dependiente } \\
\mathrm{x}=\mathrm{edad}\end{array}$ & $\mathrm{R}^{2}$ & $\begin{array}{c}\text { Valor } \\
\mathrm{p}\end{array}$ \\
\hline Densidad & 7 & $\begin{array}{l}\text { Juvenil } \\
\text { Madura }\end{array}$ & $\begin{array}{l}\mathrm{y}=345+4 \mathrm{x} \\
\mathrm{y}=403-1 \mathrm{x}\end{array}$ & 0,84 & 0,000 \\
\hline Longitud Fibras & 7 & $\begin{array}{l}\text { Juvenil } \\
\text { Madura }\end{array}$ & $\begin{array}{l}\mathrm{y}=955,2+58,25 \mathrm{x} \\
\mathrm{y}=1267,1+18,58 \mathrm{x}\end{array}$ & 0,86 & 0,000 \\
\hline Ancho Fibras & 5 & $\begin{array}{l}\text { Juvenil } \\
\text { Madura }\end{array}$ & $\begin{array}{l}\mathrm{y}=15,35+1,018 \mathrm{x} \\
\mathrm{y}=20,09+0,041 \mathrm{x}\end{array}$ & 0,85 & 0,000 \\
\hline Espesor pared & 9 & $\begin{array}{l}\text { Juvenil } \\
\text { Madura }\end{array}$ & $\begin{array}{l}\mathrm{y}=2,13+0,08 \mathrm{x} \\
\mathrm{y}=2,33+0,06 \mathrm{x}\end{array}$ & 0,82 & 0,000 \\
\hline Área de pared & 4 & $\begin{array}{l}\text { Juvenil } \\
\text { Madura }\end{array}$ & $\begin{array}{l}\mathrm{y}=47,54+0,071 \mathrm{x} \\
\mathrm{y}=49,67+0,148 \mathrm{x}\end{array}$ & 0,77 & 0,000 \\
\hline Diámetro Vasos & 4 & $\begin{array}{l}\text { Juvenil } \\
\text { Madura }\end{array}$ & $\begin{array}{l}\mathrm{y}=53,00+1,35 \mathrm{x} \\
\mathrm{y}=56,09+0,51 \mathrm{x}\end{array}$ & 0,82 & 0,000 \\
\hline $\begin{array}{l}\text { Frecuencia } \\
\text { Vasos }\end{array}$ & 5 & $\begin{array}{l}\text { Juvenil } \\
\text { Madura }\end{array}$ & $\begin{array}{l}\mathrm{y}=47,54+0,071 \mathrm{x} \\
\mathrm{y}=49,67+0,148 \mathrm{x}\end{array}$ & 0,86 & 0,000 \\
\hline
\end{tabular}

Madera Juvenil: Ecuación de ajuste del segmento anterior a la edad de transición juvenil-maduro. Madera Madura: Ecuación de ajuste del segmento posterior a la edad de transición juvenil-maduro 
Los vasos (diámetro y frecuencia) fueron, junto con el ancho de las fibras y el área de pared, los primeros parámetros en alcanzar la maduración a los 4-5 años. Luego lo hicieron la longitud de fibras y la densidad de la madera a la edad de 7 años y finalmente a los 9 años, se estabilizaron los valores de espesor de pared de las fibras.

Para densidad y longitud de fibras se aplicó el modelo de segmentación lineal para todas las alturas en el fuste (Tablas 3 y 4). De esta forma se halló la edad de transición a lo largo del fuste.

Tabla 3. Edad de transición entre madera juvenil y madura para densidad básica a las alturas de muestreo en el fuste, ecuaciones de ajuste, valor de $\mathrm{R}$ y valor $\mathrm{p}$

\begin{tabular}{cccccc}
\hline $\begin{array}{c}\text { Altura de } \\
\text { muestreo }\end{array}$ & $\begin{array}{c}\text { Edad de } \\
\text { transición } \\
\text { (años) }\end{array}$ & $\begin{array}{c}\text { Tipo de } \\
\text { madera }\end{array}$ & $\begin{array}{l}\text { Modelos } \\
\mathrm{y}=\text { var. dependiente } \\
\mathrm{x}=\text { edad }\end{array}$ & $\mathrm{R}^{2}$ & Valor $\mathrm{p}$ \\
\hline $0,3 \mathrm{~m}$ & 9 & $\begin{array}{l}\text { Juvenil } \\
\text { Madura }\end{array}$ & $\begin{array}{l}\mathrm{y}=331+3 \mathrm{x} \\
\mathrm{y}=363-2 \mathrm{x}\end{array}$ & 0,85 & 0,000 \\
\hline $1,3 \mathrm{~m}$ & 7 & $\begin{array}{l}\text { Juvenil } \\
\text { Madura }\end{array}$ & $\begin{array}{l}\mathrm{y}=345+4 \mathrm{x} \\
\mathrm{y}=403-1 \mathrm{x}\end{array}$ & 0,84 & 0,000 \\
\hline $8,2 \mathrm{~m}$ & 8 & $\begin{array}{l}\text { Juvenil } \\
\text { Madura }\end{array}$ & $\begin{array}{l}\mathrm{y}=339+3 \mathrm{x} \\
\mathrm{y}=369+2 \mathrm{x}\end{array}$ & 0,92 & 0,000 \\
\hline $15,1 \mathrm{~m}$ & 8 & $\begin{array}{l}\text { Juvenil } \\
\text { Madura }\end{array}$ & $\begin{array}{l}\mathrm{y}=353+4 \mathrm{x} \\
\mathrm{y}=379+3 \mathrm{x}\end{array}$ & 0,90 & 0,000 \\
\hline
\end{tabular}

Madera Juvenil: Ecuación de ajuste del segmento anterior a la edad de transición juvenil-maduro.

Madera Madura: Ecuación de ajuste del segmento posterior a la edad de transición juvenil-maduro

Se aprecia un descenso de la edad de transición a medida que ascendemos en el fuste para ambas variables. La madera producida por el cámbium en las partes superiores del fuste a una misma edad, presenta un grado de maduración mayor, por eso la edad de transición disminuye.

Tabla 4. Edad de transición entre madera juvenil y madura para longitud de fibras a las alturas de muestreo en el fuste, ecuaciones de ajuste, valor de $\mathrm{R}$ y valor $\mathrm{p}$

\begin{tabular}{cccccc}
\hline $\begin{array}{c}\text { Altura de } \\
\text { muestreo }\end{array}$ & $\begin{array}{c}\text { Edad de } \\
\text { transición } \\
(\text { años })\end{array}$ & $\begin{array}{c}\text { Tipo de } \\
\text { madera }\end{array}$ & $\begin{array}{c}\text { y=var. dependiente } \\
\mathrm{x}=\text { edad }\end{array}$ & $\mathrm{R}^{2}$ & Valor $\mathrm{p}$ \\
\hline $0,3 \mathrm{~m}$ & 8 & $\begin{array}{l}\text { Juvenil } \\
\text { Madura }\end{array}$ & $\begin{array}{l}\mathrm{y}=1040,1+17,60 \mathrm{x} \\
\mathrm{y}=1334,9-13,84 \mathrm{x}\end{array}$ & 0,86 & 0,000 \\
\hline $1,3 \mathrm{~m}$ & 7 & $\begin{array}{l}\text { Juvenil } \\
\text { Madura }\end{array}$ & $\begin{array}{l}\mathrm{y}=955,2+58,25 \mathrm{x} \\
\mathrm{y}=1267,1+18,58 \mathrm{x}\end{array}$ & 0,86 & 0,000 \\
\hline $4,2 \mathrm{~m}$ & 7 & $\begin{array}{l}\text { Juvenil } \\
\text { Madura }\end{array}$ & $\begin{array}{l}\mathrm{y}=1082,9+20,34 \mathrm{x} \\
\mathrm{y}=1450,3+7,77 \mathrm{x}\end{array}$ & 0,86 & 0,000 \\
\hline $8,1 \mathrm{~m}$ & 7 & $\begin{array}{l}\text { Juvenil } \\
\text { Madura }\end{array}$ & $\begin{array}{l}\mathrm{y}=1072,4+19,69 \mathrm{x} \\
\mathrm{y}=1365,7+14,79 \mathrm{x}\end{array}$ & 0,86 & 0,000 \\
\hline $15,9 \mathrm{~m}$ & 7 & $\begin{array}{l}\text { Juvenil } \\
\text { Madura }\end{array}$ & $\begin{array}{l}\mathrm{y}=1053,59+24,23 \mathrm{x} \\
\mathrm{y}=1277,1+21,78 \mathrm{x}\end{array}$ & 0,86 & 0,000 \\
\hline
\end{tabular}

Madera Juvenil: Ecuación de ajuste del segmento anterior a la edad de transición juvenil-maduro.

Madera Madura: Ecuación de ajuste del segmento posterior a la edad de transición juvenil-maduro 
Teniendo en cuenta estas dos variables podría considerarse que este clon de álamo comienza a formar madera madura entre los 7 y 9 años de edad a lo largo de todo el fuste.

La edad de demarcación entre madera juvenil y madura no fue la misma en cada propiedad. Esto indica que el control fisiológico del proceso de maduración de las variables anatómicas está determinado por el desarrollo del tronco y de la copa durante el crecimiento del árbol (Zobel y Sprague 1998). Los últimos parámetros que alcanzaron la maduración fueron la densidad, longitud y espesor de pared de las fibras, y dicha información puede ser importante para definir edades de rotación de una plantación para los diferentes destinos industriales (triturado o aserrado).

Aplicando el mismo modelo estadístico Matyas y Peszlen (1997) hallaron edades de transición muy semejantes a las de este trabajo, estudiando 3 clones de Populus $x$ euroamericana de 10 y 14 años a una única altura en el fuste, en dos sitios de Hungría. De la misma forma, hallaron que para diferentes propiedades anatómicas las edades de transición variaron entre 4-6 años para ancho de fibras, área de pared y diámetro de vasos, y entre 9-10 años para longitud de fibras. Demostraron también que el sitio influyó en la edad de maduración (pero no en el orden de maduración) ya que los valores medios se modificaron al determinarlos en árboles implantados en otro sitio.

Teniendo en cuenta la densidad como propiedad, Bendtsen y Senft (1986) determinaron que el punto de demarcación entre madera juvenil y madura en Populus deltoides de 30 años en EE.UU, se encontraba alrededor de los 17 a 18 años. Para longitud de fibras y ángulo microfibrillar los valores se estabilizaron dos años más tarde.

\section{Propiedades de la madera juvenil y madura}

Los valores promedio por tipo de madera juvenil o madura calculados para la altura de $1,3 \mathrm{~m}$ se presentan en la tabla 5. Se tomó como edad límite entre leños a las edades de transición obtenidas por las regresiones (Tabla 2). Las edades de transición variaron según la variable considerada entre los 4 , 5,7 y 9 años.

Tabla 5. Valores promedio de las propiedades en madera juvenil y madera madura a la altura de $1,3 \mathrm{~m}$ en el fuste.

\begin{tabular}{|c|c|c|c|c|c|c|c|c|}
\hline $\begin{array}{l}\text { Tipo de } \\
\text { madera }\end{array}$ & $\begin{array}{l}\mathrm{AB} \mathrm{c \textrm {cm } ^ { 2 }} \\
\text { (4-9 años) }\end{array}$ & $\begin{array}{c}\mathrm{D} \\
\mathrm{kg} \mathrm{m}^{-3}\end{array}$ & $\begin{array}{c}\mathrm{LF} \\
10^{-3} \mathrm{~mm}\end{array}$ & $\begin{array}{c}\mathrm{AF} \\
10^{-3} \mathrm{~mm}\end{array}$ & $\begin{array}{c}\mathrm{EF} \\
10^{-3} \mathrm{~mm}\end{array}$ & $\mathrm{AP}$ & $\begin{array}{c}\mathrm{DV} \\
10^{-3} \mathrm{~mm}\end{array}$ & $\begin{array}{c}\mathrm{FV} \\
\mathrm{n}^{0} \mathrm{~mm}^{-1}\end{array}$ \\
\hline Juvenil & $55-424$ & $350 a^{*}$ & $1190 \mathrm{a}$ & $18,62 \mathrm{a}$ & $2,54 \mathrm{a}$ & $48 \mathrm{a}$ & 58,76 a & $80 \mathrm{~b}$ \\
\hline Madura & 949-580 & $380 \mathrm{~b}$ & $1490 \mathrm{~b}$ & $20,55 b$ & $3,16 \mathrm{~b}$ & $50 \mathrm{a}$ & $72,05 \mathrm{~b}$ & $57 \mathrm{a}$ \\
\hline
\end{tabular}

AB: área basal, D: densidad, LF: longitud de fibras, AF: ancho de fibras, EF: espesor pared de fibras, AP: área de pared, DV: diámetro de vasos, FV: frecuencia de vasos.

*Las letras se leen verticalmente. Letras distintas denotan diferencias significativas según prueba de Tukey $(\mathrm{P}<0,05)$. 
Las propiedades de la madera juvenil a la altura de 1,3 $\mathrm{m}$ fueron significativamente inferiores, según el ANOVA, para todas las variables $(\mathrm{p}<0,05)$ excepto el área de pared $\left(\mathrm{F}_{1,15}=1,38 ; \mathrm{p}=0,257\right)$. La madera juvenil presentó menor densidad, menor morfometría de fibras (ancho, espesor y largo), menor diámetro de vasos y mayor frecuencia de los mismos (Tabla 5).

La densidad básica y longitud de fibras de la madera juvenil calculadas como promedio de todas las alturas (árbol completo) y con una edad de transición de 8 años, indican que la madera juvenil es inferior en calidad a lo largo de todo su cilindro central (Tabla 6, prueba de Tukey).

Tabla 6. Valores promedio de las alturas muestreadas en el fuste para la densidad básica y longitud de fibras en madera juvenil y madera madura (edad de transición 8 años) de acuerdo al volumen de madera promedio por árbol

\begin{tabular}{cccc}
\cline { 2 - 3 } Tipo de madera & Volumen & Densidad & $\begin{array}{c}\text { Longitud } \\
\text { Fibras } \\
10^{-3} \mathrm{~mm}\end{array}$ \\
\hline Juvenil & 0,62 & $366 \mathrm{a}^{*}$ & $1215,64 \mathrm{a}$ \\
Madura & 0,56 & $394 \mathrm{~b}$ & $1500,94 \mathrm{~b}$ \\
\hline
\end{tabular}

*Las letras se leen verticalmente. Letras distintas denotan diferencias significativas según prueba de Tukey $(\mathrm{P}<0,05)$. 
Para los árboles de 17 años muestreados, el cilindro central juvenil representó el 52\% del volumen total cosechado $(1,18 \mathrm{~m} 3)$.

Para turnos de rotación cortos (10 años), como son los usados en la industria papelera en Argentina, la proporción de madera juvenil podría considerarse del $87 \%$ del volumen comercial del árbol según las tendencias que marcan este estudio. Los turnos de rotación de 17 años o más son utilizados para la industria del aserrado o del debobinado, y por lo tanto, se esperaría obtener una mayor proporción de madera madura $(50 \%)$ de mayor calidad.

\section{CONCLUSIONES}

Los resultados obtenidos marcan una tendencia que debe ser analizada profundamente para otros clones de álamos y sitios de implantación.

En este sentido, este estudio presenta una línea de exploración con posibilidades de aplicación para el buen aprovechamiento de la madera según calidades y los requisitos de la industria consumidora.

El pasaje de madera juvenil a madura no se produce a la misma edad según la propiedad que se considere. En el clon $P$. deltoides 'Australia 129/60' la tendencia preliminar indica que la edad varía entre los 4 y 9 años según se trate de los vasos, la longitud de fibras, la densidad de la madera o el espesor de pared de fibras.

Independientemente de la edad de transición y la propiedad elegida para su análisis, la madera juvenil presentó calidad inferior en todos los casos. Esto podría suponer que, dependiendo de la industria consumidora (triturado, aserrado o laminado), la edad de cosecha del rodal (turno) podría manejarse para influir en las propiedades del árbol completo considerando la proporción final de madera juvenil y madura.

\section{AGRADECIMIENTOS}

A Papel Prensa S.A., por facilitar el acceso al establecimiento y financiar el muestreo.

\section{REFERENCIAS}

Achinelli, F.G. 2006. Silvicultura de álamos y sauces en la Pampa Húmeda. Actas de las I Jornadas Argentinas de Salicáceas, Buenos Aires, Argentina. pp21-36.

Bendtsen, B.A.; Senft, J. 1986. Mechanical and anatomical properties in individual growth rings of plantation-grown eastern cottonwood and loblolly pine. Wood and Fiber Science 18(1): 23-38.

Bhat, K.M.; Priya, P.B.; Rugmini, P. 2001. Characterisation of juvenile wood in teak. Wood Science and Technology 34 (6): 517-532.

Borodowski, E. 2006. Álamos y sauces en el Delta del Paraná: situación del sector y silvicultura. Actas de las I Jornadas Argentinas de Salicáceas, Buenos Aires, Argentina. pp. 61-70.

DeBell, D.S.; Singleton, R.; Harrington, C.A.; Gartner, B.L. 2002. Wood density and fiber length in young Populus stem: relation to clone, age, growth rate, and pruning. Wood and Fiber Science 34 (4): 529-539. 
Franklin, G.L. 1945. Preparation of thin sections of synthetic resins and wood-resin composites, and a new macerating method for wood. Nature 155 (3924): 51.

Instituto Argentino de Normalización. 1973. Maderas. Método de determinación de la densidad aparente. Norma IRAM 9544. Buenos Aires, Argentina.

Matyás, C.; Peszlen, I. 1997. Effect of age on selected wood quality traits of poplar clones. Silvae Genetica 46(2-3): 64-72.

Monteoliva, S.; Senisterra, G. 2008. Efecto del sitio, el origen y el clon sobre el crecimiento y propiedades de la madera de Populus. Revista de Investigación Agraria, Sistema y Recursos Forestales 17 (3): 261-270.

SAGPyA. Secretaria de Agricultura, Ganadería, Pesca y Alimentos. 2004. Evolución y perspectivas del cultivo de salicáceas en la pampa húmeda argentina. Revista SAGPyA Forestal 32: 14-24.

Tasissa, G.; Burkhart, H.E. 1998. Juvenile-mature wood demarcation in loblolly pine trees. Wood and Fiber Science 30 (2): 118-127.

Zobel, B.J.; Jett, J.B. 1995. Genetics of wood production. Springer-Verlag, Berlín. 337pp.

Zobel, B.J.; Sprague, J.R. 1998. Juvenile wood in forest trees. Springer-Verlag, Berlín. 308pp. 